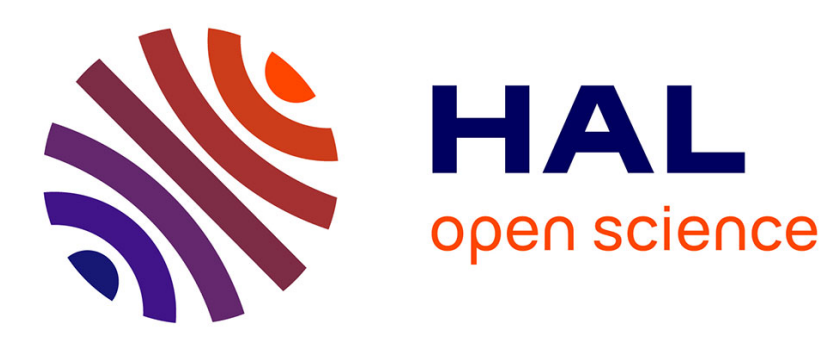

\title{
Contribution à l'histoire de l'épidémiologie des facteurs de risque \\ Élodie Giroux
}

\section{To cite this version:}

Élodie Giroux. Contribution à l'histoire de l'épidémiologie des facteurs de risque. Revue d'Histoire des Sciences, 2011, 64 (2), pp.219-224. 10.3917/rhs.642.0219 . halshs-00854345

\section{HAL Id: halshs-00854345 \\ https://shs.hal.science/halshs-00854345}

Submitted on 23 Apr 2020

HAL is a multi-disciplinary open access archive for the deposit and dissemination of scientific research documents, whether they are published or not. The documents may come from teaching and research institutions in France or abroad, or from public or private research centers.
L'archive ouverte pluridisciplinaire HAL, est destinée au dépôt et à la diffusion de documents scientifiques de niveau recherche, publiés ou non, émanant des établissements d'enseignement et de recherche français ou étrangers, des laboratoires publics ou privés. 


\section{CONTRIBUTION À L'HISTOIRE DE L'ÉPIDÉMIOLOGIE DES FACTEURS DE RISQUE}

Élodie Giroux

Armand Colin / Dunod | « Revue d'histoire des sciences »

2011/2 Tome 64 | pages 219 à 224

ISSN 0151-4105

Article disponible en ligne à l'adresse :

http://www.cairn.info/revue-d-histoire-des-sciences-2011-2-page-219.htm

\section{Pour citer cet article :}

Élodie Giroux, «Contribution à l'histoire de l'épidémiologie des facteurs de risque », Revue d'histoire des sciences 2011/2 (Tome 64), p. 219-224.

DOI 10.3917/rhs.642.0219

Distribution électronique Cairn.info pour Armand Colin / Dunod.

(C) Armand Colin / Dunod. Tous droits réservés pour tous pays.

La reproduction ou représentation de cet article, notamment par photocopie, n'est autorisée que dans les limites des conditions générales d'utilisation du site ou, le cas échéant, des conditions générales de la licence souscrite par votre établissement. Toute autre reproduction ou représentation, en tout ou partie, sous quelque forme et de quelque manière que ce soit, est interdite sauf accord préalable et écrit de l'éditeur, en dehors des cas prévus par la législation en vigueur en France. Il est précisé que son stockage dans une base de données est également interdit. 


\section{DOSSIER}

\section{Contribution à l'histoire de l'épidémiologie des facteurs de risque Élodie GIROUX*}

L'épidémiologie est aujourd'hui communément définie comme l'étude de la fréquence des pathologies et de la distribution des états de santé dans les populations humaines, et de leurs déterminants. Son histoire, bien que récente, commence à faire l'objet d'un nombre significatif d'études spécifiques, à la fois par ses acteurs et par des historiens ${ }^{1}$. Ce dossier se concentre sur les changements survenus au milieu du $\mathrm{Xx}^{\mathrm{e}}$ siècle, souvent considérés comme une étape essentielle de son histoire : c'est à ce moment-là qu'elle aurait acquis, en particulier aux États-Unis et en Grande-Bretagne, un statut de discipline scientifique. Un certain consensus tend à se dégager pour souligner l'émergence d'un " nouveau style d'épidémiologie ${ }^{2}$ » diversement caractérisé comme " épidémiologie moderne ${ }^{3}$ », " épidémiologie des maladies chroniques ", " épidémiologie du risque ${ }^{4}$ » ou encore "épidémiologie des facteurs de risque ${ }^{5}$ ». Cette "nouvelle épidémiologie ${ }^{6}$ » aurait contribué au développement d'une

* Élodie Giroux, maître de conférences, Faculté de philosophie, Université Jean-Moulin Lyon-3, 1, rue de I'Université, 69007 Lyon et Institut de recherches philosophiques de Lyon, 18, rue Chevreul, 69007 Lyon.

E-mail : elodie.giroux@univ-lyon3.fr

1 - Voir bibliographie indicative.

2 - Luc Berlivet, "Association or causation? ": The debate on the scientific status of risk factor epidemiology (1947 - c.1965), Clio medica, 75 (2005), 39-74.

3 - "Épidémiologie moderne " est le titre du manuel d'un des épidémiologistes les plus influents du Xx ${ }^{\mathrm{e}}$ siècle, Kenneth Rothman : Modern epidemiology (Boston : Little, Brown and Company, 1986). Toutefois, selon la périodisation proposée par Alfredo Morabia, il pourrait être plus juste de distinguer une phase appelée " épidémiologie classique » (19451980), et dont il est donc ici question, de l'« épidémiologie moderne », qui correspondrait plus précisément aux années quatre-vingt et suivantes. Voir Alfredo Morabia (ed.), A history of epidemiologic methods and concepts (Basel : Birhäuser, 2004), 117-124.

4 - Luc Berlivet, Déchiffrer la maladie, in Jean-Pierre Dozon et Didier Fassin (dir.), Critique de la santé publique : Une approche anthropologique (Paris : Balland, 2001), 95.

5 - Voir par exemple Berlivet, art. cit. in n. 2 ; Mervyn Susser, Does risk factor epidemiology put epidemiology at risk? Peering into the future, Journal of epidemiologic community health, 52 (1998), 608-611.

6 - John E. Gordon, Epidemiology : Old and new, Journal of the Michigan State Medical Society, 49 (1950), 194-199. 
approche quantitative et probabiliste de la maladie à partir de ses facteurs de risque, principalement individuels ${ }^{7}$. Cette approche joue aujourd'hui un rôle déterminant, aussi bien en médecine que dans le domaine de la santé publique, pour la prévention de nombreuses maladies chroniques et multifactorielles.

Pour caractériser ces changements de l'épidémiologie, on a souvent insisté sur un renouvellement de ses objets (extension aux maladies chroniques) et de ses méthodes : en particulier, la formalisation de deux dispositifs d'enquête d'observation qui permettent l'identification des facteurs de risque, l'étude castémoins et l'étude de cohorte, et le recours à de nouvelles techniques d'analyse statistique qui garantissent une meilleure objectivité et solidité de l'inférence. D'une étude principalement descriptive des épidémies de maladies infectieuses et transmissibles, l'épidémiologie serait devenue le lieu privilégié de l'analyse statistique des étiologies complexes de tout type de pathologie. L'intégration et l'adaptation des techniques issues de la statistique mathématique, principalement développée dans les années trente par des Britanniques comme Ronald Fisher et Karl Pearson, auraient contribué à ces principales modifications.

Toutefois, sur ce prétendu tournant de l'épidémiologie, un grand nombre de questions reste à explorer. Tout d'abord, quelle est la véritable nature des ruptures avec l'épidémiologie du début du $\mathrm{Xx}^{\mathrm{e}}$ siècle ? Les origines de son institutionnalisation et de sa constitution comme discipline remontent au milieu du $\mathrm{XIX}^{\mathrm{e}}$ siècle, et $\mathrm{c}^{\prime}$ est dès les années vingt qu'elle devient une discipline académique. En Grande-Bretagne, Major Greenwood - qui s'était formé en statistique auprès de Pearson - fut nommé en 1930 professeur d'épidémiologie au département d'épidémiologie et de statistiques vitales de l'école d'hygiène et de médecine tropicale de Londres, et il publia en 1935 l'un des premiers manuels d'épidémiologie ${ }^{8}$. Aux États-Unis, Wade Hampton Frost fut, en 1922, le premier titulaire d'une chaire d'épidémiologie dans le département d'épidémiologie et d'administration de

7 - William Rothstein, Public health and the risk factor : A history of an uneven medical revolution (Rochester: University of Rochester Press, 2003).

8 - Major Greenwood, Epidemics and crowd-diseases : An introduction to the study of epidemiology (New York : Macmillan, 1935). 
santé publique à l'école d'hygiène et de santé publique de I'université Johns-Hopkins à Baltimore.

Par ailleurs, I'intérêt porté aux maladies chroniques multifactorielles, principalement les cancers et les maladies cardio-vasculaires, accompagnait la transition épidémiologique dans les pays les plus développés, dont les effets se firent ressentir dès le début du $\mathrm{xx}^{\mathrm{e}}$ siècle. Surtout, des maladies à la fois infectieuses et chroniques telles que la tuberculose et la syphilis firent l'objet d'analyses étiologiques dans l'épidémiologie du premier tiers du $x x^{\mathrm{e}}$ siècle, qui furent l'occasion d'innovations méthodologiques fondamentales pour le développement ultérieur de l'épidémiologie des facteurs de risque ${ }^{9}$. En outre, des travaux menés sur les épidémies de maladies infectieuses dans le cadre de l'épidémiologie expérimentale, en particulier en GrandeBretagne, avaient déjà conduit au développement de modèles mathématiques multifactoriels et interactionnistes ${ }^{10}$.

On peut aussi s'interroger sur la part de responsabilité de l'épidémiologie dans l'émergence de l'approche quantitative et probabiliste de la maladie que véhicule la notion de risque. Ne convient-il pas de situer cette approche dans un contexte épistémologique, mais aussi socioculturel, politique et économique plus global qu'une histoire trop internalisée de l'épidémiologie tendrait à négliger ${ }^{11}$ ? En outre, le développement de cette discipline originellement associée aux statistiques sanitaires et à la santé publique semble lié au pays dans lequel elle s'inscrit, mais aussi à la pathologie à laquelle elle s'intéresse. Quelle est dès lors l'unité de ce qu'on désigne par "épidémiologie des facteurs de risque " ? II reste aussi à mieux élucider pourquoi on a assisté à une telle suprématie anglo-américaine alors qu'au $\mathrm{XIX}^{\mathrm{e}}$ siècle, la Prusse mais aussi la France par exemple avaient

9 - Voir notamment les travaux de Frost. Kenneth F. Maxcy (ed.), Papers of Wade Hampton Frost : A contribution to epidemiological method (New York : Commonwealth Fund, 1941).

10 - Olga Amsterdamska, Achieving disbelief : Thought styles, microbial variation, and American and British epidemiology, 1900-1940, Studies in history and philosophy of biological and biomedical sciences, 35 (2004), 483-507.

11 - Le rôle des compagnies d'assurance sur la vie a été bien mis en évidence. Voir notamment Rothstein, op. cit. in n. 7. Pour relativiser la place de l'épidémiologie dans le développement et l'introduction de cette approche des maladies, voir l'article de Robert Aronowitz dans ce dossier, mais aussi l'ouvrage de Jeremy Greene : Prescribing by numbers : Drug and the definition of disease (Baltimore : Johns Hopkins University, 2007). 
une avance notable dans le développement de statistiques sanitaires et $d^{\prime}$ une forme de santé publique ${ }^{12}$.

Ce dossier apporte des éléments de réponses à ces questions. L'un des principaux objectifs est de mieux cerner les continuités et les ruptures introduites par l'épidémiologie des facteurs de risque en s'intéressant aux méthodes d'enquête d'observation qui lui sont associées et à son traitement de la causalité et de la multifactorialité des maladies. En effet, la causalité s'est rapidement trouvée au cour du questionnement de cette épidémiologie avec la nécessité d'interpréter le statut causal des associations statistiques identifiées par ces enquêtes. Ce dossier se concentre par ailleurs sur les pathologies emblématiques du développement de ce nouveau style d'épidémiologie, le cancer du poumon et les maladies cardio-vasculaires, tout en ayant le souci de montrer la coloration spécifique des épidémiologies correspondantes.

Les historiens de l'épidémiologie du risque se sont principalement intéressés à l'épidémiologie du cancer du poumon au point de rendre ces deux types d'épidémiologie presque synonymes ${ }^{13}$. En effet, les enquêtes anglaises et américaines menées au tournant des années quarante et cinquante sur l'étiologie de cette maladie sont souvent considérées comme ayant conduit à la formalisation des principales caractéristiques de cette nouvelle épidémiologie. La légitimation de l'épidémiologie comme discipline scientifique était au cœur de la controverse sur le statut causal de I'association statistique entre tabagisme et cancer du poumon (1950-1964) ${ }^{14}$. Deux articles de ce dossier apportent un éclairage original sur cette controverse. En comparant ces études angloaméricaines avec les études allemandes menées dans le contexte du régime nazi, Alfredo Morabia met en lumière l'importance du contexte politique dans l'émergence de l'épidémiologie du risque et de sa légitimation scientifique. En l'occurrence, le contexte démocratique des études anglo-américaines a contribué à la for-

12 - Voir par exemple, pour la France, William Coleman, Death is a social disease : Public health and political economy in early industrial France (Wisconsin : University of Wisconsin Press, 2002).

13 - Berlivet, art. cit. in n. 2

14 - Ibid. ; Mark Parascandola, Skepticism, statistical methods, and the cigarette : A historical analysis of a methodological debate, Perspectives in biology and medicine, 47/2 (2004), 246-261. 
malisation rigoureuse de protocoles d'enquête d'observation et au développement de critères pour l'inférence causale. Cette " épidémiologie politique » permet en outre de comprendre une forme de parenthèse du développement de l'épidémiologie allemande à cette époque. Mark Parascandola s'intéresse à l'évolution du concept de causalité dans l'épidémiologie des facteurs de risque. La comparaison avec ce qui se joue alors dans d'autres disciplines à la même époque, en particulier la physique quantique et la biologie, permet de situer la spécificité des apports et du traitement qu'en fait l'épidémiologie. Tous les épidémiologistes furent loin d'adopter un concept probabiliste et de renoncer au modèle traditionnel de la causalité spécifique véhiculé par la bactériologie et le laboratoire. On observe par ailleurs une tension entre visée d'action et visée de recherche, qui apparaît alors comme constitutive de l'épidémiologie dans son traitement de la causalité.

De son côté, l'épidémiologie cardio-vasculaire a été moins étudiée pour son rôle dans le développement de l'« épidémiologie moderne ", étant plus généralement appréhendée comme une sous-discipline de cette dernière ${ }^{15}$. Elle recèle pourtant des spécificités et, pour Robert Aronowitz, c'est plus précisément en son sein qu'est née ce qu'il appelle I' « approche facteurs de risque " des maladies ${ }^{16}$. Les deux autres articles de ce dossier lui sont consacrés et portent sur la fameuse étude de cohorte qui commença en 1947 à Framingham, une ville près de Boston, et joua un rôle central dans le développement et la diffusion de la notion de "facteur de risque » et des techniques d'analyse épidémiologique associées à cette notion. Aronowitz analyse et situe plus précisément ce rôle dans le contexte global de l'évolution de la médecine et de la cardiologie américaines de l'époque. L'article d'Élodie Giroux porte sur les origines du plan de l'étude prospective de cohorte et met en évidence, à

15 - Berlivet, art. cit. in n. 2 : «Bien que les études épidémiologiques sur le cancer et celles sur les maladies cardiovasculaires comme celle de Framingham commencèrent entre 1947 et 1949, les études sur l'étiologie du cancer du poumon sont décrites comme le moment décisif dans l'émergence de l' "épidémiologie moderne » alors que l'étude de Framingham est généralement considérée, de manière plus restreinte, comme le point de départ de l'épidémiologie cardiovasculaire - c'est-à-dire d'une sous-discipline à l'intérieur du cadre principal. »

16 - Robert Aronowitz, La construction sociale des facteurs de risque des maladies coronariennes, in Id., Les Maladies ont-elles un sens?, trad. de I'anglais [1998] (Paris : Synthélabo, 1999), 225. 
partir d'une comparaison avec l'épidémiologie du cancer du poumon, la spécificité de la contribution de l'épidémiologie cardio-vasculaire américaine à cette méthode d'enquête.

Remerciements. Pour terminer, je souhaite vivement remercier les trois contributeurs de ce dossier qui m'ont accordé leur confiance. Par ailleurs, ce dossier n'aurait jamais vu le jour sans la persévérance et les encouragements de Jean Gayon ni sans le soutien indéfectible de Jean-Claude Dupont. Qu'ils soient tous deux très chaleureusement remerciés. 